\title{
INFLUÊNCIA DO MATERIAL E VOLUME DO PORTA-AMOSTRA NA DETERMINAÇÃO TERMOGRAVIMÉTRICA DO TEOR DE CINZAS EM MEL
}

\author{
Maria Lurdes Felsner*, Roy Edward Bruns e Jivaldo do Rosário Matos \\ Instituto de Química, Universidade de São Paulo, Av. Prof. Lineu Prestes, 748, 05508-900 São Paulo - SP \\ Cristiane Bonaldi Cano \\ Seção de Bebidas, Instituto Adolfo Lutz, Av. Dr. Arnaldo, 355, 01246-902 São Paulo - SP \\ Lígia Bicudo Almeida-Muradian \\ Faculdade de Ciências Farmacêuticas, Universidade de São Paulo, Av. Prof. Lineu Prestes, 580, 05508-900 São Paulo - SP
}

Recebido em 23/4/04; aceito em 24/11/04; publicado na web em 13/4/05

\begin{abstract}
INFLUENCE OF MATERIAL AND VOLUME OF THE SAMPLE HOLDER ON THE THERMOGRAVIMETRIC DETERMINATION OF ASH CONTENTS IN HONEY. The results suggest that the two variables studied are significant and that they may be independently optimized. The material of the sample holder interferes with the incineration process only due to the amount of heat transfered. The sample holder volume aids diffusion of the atmosphere to the honey sample, minimizing the foaming effect. According to the results, for the thermogravimetric analysis of ash content in honey, sample holders of platinum or alumina of $150 \mu \mathrm{L}$ are indicated.
\end{abstract}

Keywords: honey; thermogravimetry; factorial design

\section{INTRODUÇÃO}

A termogravimetria (TG) é uma das técnicas de análise térmica em que as variações de massa da amostra (ganho ou perda) são monitoradas como uma função da temperatura e/ou tempo, enquanto a substância é submetida a um programa controlado de temperatura, sob uma atmosfera especificada ${ }^{1-3}$. Quando o aquecimento do produto envolve perdas de massa, podem ser estudadas características de secagem dos alimentos a partir da eliminação de umidade, bem como características dos processos de tostagem e torrefação. No entanto, quando o aquecimento do alimento resulta em ganho de massa durante os ensaios termogravimétricos, esta técnica é uma ferramenta valiosa para estudos da estabilidade oxidativa de óleos e gorduras ${ }^{1,4-5}$.

A termogravimetria tem sido empregada também para determinação dos teores de cinzas de alimentos, como café torrado, leite em pó, amido e farinha, mostrando-se vantajosa em relação aos métodos convencionais, por ser realizada em menores tempos de análise, utilizando menores massas de amostra, além de dispensar o pré-tratamento da amostra ${ }^{6-12}$. Todavia, encontram-se poucos trabalhos na literatura que adotam esta técnica para análise dos teores de cinzas em alimentos líquidos e viscosos, tais como xaropes de açúcar, geléias de frutas, mel, melaço de cana, entre outros. Estes alimentos apresentam, normalmente, efeitos de borbulhamento e projeção de amostra durante o aquecimento, necessitando, nos métodos convencionais, de um pré-tratamento da amostra ${ }^{12}$. Estes resultados encorajam investigações da termogravimetria para determinação dos teores de cinzas em amostras de méis.

Para o desenvolvimento de um método termogravimétrico para a análise dos teores de cinzas nesse produto torna-se necessário otimizar as condições de análise. Os fatores que podem ser controlados para desenvolver um método analítico são taxa de aquecimento, atmosfera do forno, composição e volume do porta-amostra e massa de amostra ${ }^{2,3}$. Das variáveis citadas acima, normalmente a primeira a ser estudada é o cadinho. Deve-se salientar que

*e-mail: mlfelsner@yahoo.com.br a geometria, o tamanho e o material de construção do porta-amostra ou cadinho têm um efeito bastante pronunciado na curva TG/ DTG. O tipo de cadinho usado depende do tamanho e natureza da amostra e da temperatura máxima a ser utilizada no experimento ${ }^{3}$.

A forma geométrica do cadinho (volume) é também importante, porque pode afetar a interação da atmosfera gasosa com a amostra. Um cadinho raso ou mais amplo permitirá a livre difusão da atmosfera até a amostra e a eliminação dos voláteis libertados do meio reacional, enquanto que um cadinho estreito, fôrma alta, pode restringir o fluxo de gás ${ }^{2,3}$.

Em vista disso, neste trabalho será realizado um estudo da influência do material e do volume do cadinho na incineração de amostras de mel pela termogravimetria, aplicando-se um planejamento fatorial $2^{2}$.

\section{PARTE EXPERIMENTAL}

\section{Materiais}

Uma amostra de mel de eucalipto que foi armazenada em recipiente plástico a $-18^{\circ} \mathrm{C}$ até a realização dos ensaiose cadinhos de platina e alumina com volumes de 60 e $150 \mu \mathrm{L}$, respectivamente.

\section{Método}

\section{Termogravimetria}

As curvas TG/DTG da amostra de mel de eucalipto usada no planejamento fatorial foram obtidas usando-se uma termobalança TGA-51, de marca Shimadzu, atmosfera dinâmica de ar sintético $(50 \mathrm{~mL} / \mathrm{min})$ e massas de $25,0 \pm 2,0 \mathrm{mg}$. Para o planejamento fatorial, além das condições experimentais citadas acima, foi usada uma taxa de aquecimento de $10^{\circ} \mathrm{C} / \mathrm{min}$.

\section{Planejamento fatorial}

A influência do material e do volume do porta-amostra (cadinho) 
nos teores de cinzas de amostras de mel foi investigada por um planejamento fatorial $2^{2}$, cada fator sendo estudado em dois níveis $^{13,14}$. As curvas TG/DTG em duplicata foram obtidas em ordem aleatória para todas as combinações dos níveis dos fatores apresentados na Tabela 1.

Tabela 1. Fatores e níveis do planejamento fatorial $2^{2}$ para a determinação do teor de cinzas em amostras de mel pela termogravimetria

\begin{tabular}{|c|c|c|}
\hline Fatores & Nível (-) & Nível (+) \\
\hline $\begin{array}{l}\text { (1) Material do } \\
\text { porta-amostra }\end{array}$ & Platina & Alumina \\
\hline $\begin{array}{l}\text { (2) Volume do } \\
\text { porta-amostra }(\mu L)\end{array}$ & $60(\phi=4 \mathrm{~mm})$ & $150(\phi=7 \mathrm{~mm})$ \\
\hline
\end{tabular}

${ }^{a}$ os cadinhos possuíam alturas similares e diâmetros internos $(\phi)$ diferentes: cadinho de $60 \mu \mathrm{L}$ com $\phi=4 \mathrm{~mm}$

Os efeitos dos fatores dos planejamentos fatoriais foram calculados por:

$\mathrm{E}_{\mathrm{f}}=\left(\overline{\mathrm{R}}^{+}\right)-\left(\overline{\mathrm{R}}^{-}\right)$

em que $\overline{\mathrm{R}}^{+}$e $\overline{\mathrm{R}}^{-}$são as diferenças das médias dos níveis (+) e (-), respectivamente, dos fatores envolvidos.

Os efeitos das variáveis nos teores de cinzas foram testados para a significância estatística no nível de $95 \%$ pelo cálculo do erro padrão. A análise estatística dos dados foi realizada empregando-se o "Statistica Industrial System"15.

\section{RESULTADOS E DISCUSSÃO}

A curva TG/DTG da amostra de mel estudada no planejamento fatorial evidenciou um processo de decomposição térmica complexo, envolvendo várias etapas que iniciam à temperatura ambiente e finalizam próximo a $600{ }^{\circ} \mathrm{C}$. De acordo com Felsner ${ }^{16}$, o produto final, termicamente estável a $600{ }^{\circ} \mathrm{C}$, corresponde às cinzas da amostra de mel.

Os resultados do planejamento fatorial são apresentados na Tabela 2 e os efeitos calculados estão listados na Tabela 3.

Tabela 2. Efeitos calculados e seus desvios-padrão para o planejamento fatorial $2^{2}$ para a determinação do teor de cinzas pela termogravimetria

\begin{tabular}{lcc}
\hline $\begin{array}{l}\text { Material porta- } \\
\text { amostra }\end{array}$ & $\begin{array}{c}\text { Volume porta- } \\
\text { amostra }(\mu \mathrm{L})\end{array}$ & $\begin{array}{c}\text { Teores médios de } \\
\text { cinzas } \pm \text { desvio-padrão }\end{array}$ \\
\hline Platina (-) & $60(-)$ & $1,061 \pm 0,120$ \\
Alumina (+) & $60(-)$ & $2,098 \pm 0,305$ \\
Platina (-) & $150(+)$ & $0,538 \pm 0,125$ \\
Alumina (+) & $150(+)$ & $1,560 \pm 0,124$ \\
\hline
\end{tabular}

a desvio- padrão calculado a partir da realização de duas curvas TG/DTG da amostra de mel

A partir da análise do teste- $t$ e teste- $F$ no nível de confiança de $95 \%$ foram consideradas como significativas todas as variáveis do planejamento fatorial (material e volume do porta-amostra), mas não foi observado um efeito de interação significativo entre as variáveis investigadas (Tabela 3 e Figura 1). Isto significa que as variáveis material e volume do porta-amostra podem ser otimizadas de forma independente.
Tabela 3. Efeitos médios calculados e seus erros padrão para o planejamento fatorial $2^{2}$, para determinação do teor de cinzas em mel por termogravimetria

\begin{tabular}{lc}
\hline Efeitos & Estimativa \pm erro padrão ${ }^{\text {a }}$ \\
\hline Média global & $1,314 \pm 0,065$
\end{tabular}

Efeitos principais:

Material do porta-amostra (1) $\quad 1,030 \pm 0,131$

Volume do porta-amostra (2) $\quad-0,531 \pm 0,131$

Interação de dois fatores:

(1) x (2) $\quad-0,008 \pm 0,131$

a o erro padrão dos efeitos foi calculado a partir dos desvios padrão apresentados na Tabela 2.

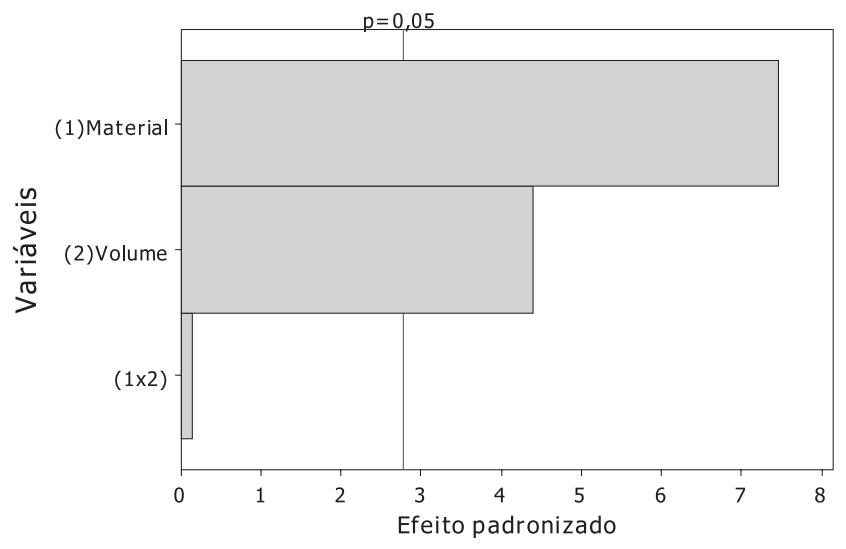

Figura 1. Gráfico de Pareto para os efeitos padronizados do planejamento fatorial, nível de confiança de $95 \%$.

A mudança de nível da variável material do cadinho, de platina para alumina, resultou em um aumento nos teores médios de cinzas em aproximadamente $1,0 \%$, independente do nível da variável volume do cadinho (Figura 2). Isto pode ser explicado pelas diferenças nas características de transferência de calor de cada tipo de material do cadinho. A platina apresenta maior condutividade térmica em relação à alumina propiciando, assim, um aumento na transferência de calor entre a amostra e o porta-amostra. Isto faz com que a eliminação da matéria orgânica seja mais rápida nos cadinhos de platina, embora estes possam favorecer, também, a formação de pontos de carvão na incineração. No entanto, cadinhos com maior volume $(150 \mu \mathrm{L})$, independentemente do tipo do material do porta-amostra (platina ou alumina) adotado, mostraram teores médios de cinzas aproximadamente $0,5 \%$ mais baixos que os encontrados em cadinhos de menor volume $(60 \mu \mathrm{L})$. Este fato pode estar relacionado às características da amostra analisada. Este tipo de produto apresenta normalmente altas concentrações de açúcar e água, que são responsáveis pelo efeito de borbulhamento observado durante o aquecimento, o qual proporciona um aumento considerável do volume da amostra no cadinho. Desta forma, pode-se sugerir que o aumento do volume do cadinho facilita a difusão da atmosfera oxidante na amostra e favorece a eliminação da matéria orgânica (Figura 2).

Além dos efeitos principais não foram observados efeitos de interação de dois fatores significativos entre as variáveis estudadas (Tabela 3), ou seja, os fatores podem ser analisados separadamente, pois seus efeitos não dependem dos níveis das outras variáveis (Figura 3). 


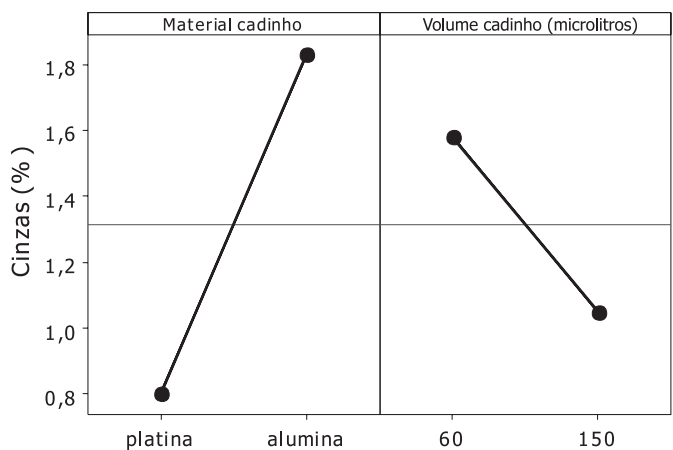

Figura 2. Diagrama para interpretação dos efeitos do planejamento fatorial $2^{2}$ para a análise do teor de cinzas em mel pela termogravimetria

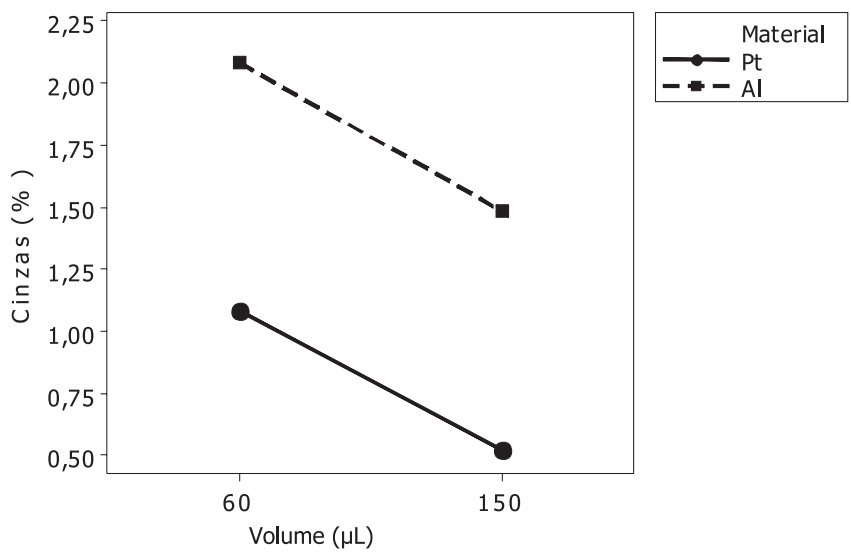

Figura 3. Gráfico do efeito de interação entre material e volume do portaamostra para o planejamento fatorial $2^{2} .(P t=$ platina $e \mathrm{Al}=$ alumina $)$

Os teores médios de cinzas obtidos pela termogravimetria neste estudo (Tabela 2) ainda são muito superiores aos encontrados normalmente em méis (inferiores ou iguais a $0,6 \%)^{17,18}$. Isto demonstra que outros fatores de natureza instrumental, ou relacionados às características da amostra, deveriam ser investigados por outros planejamentos experimentais antes desta metodologia poder ser aplicada a amostras de méis para a determinação do teor de cinzas.

\section{CONCLUSÕES}

Os resultados do planejamento fatorial sugeriram que as variáveis investigadas material e volume do porta-amostra (cadinho) influenciam no processo de incineração de amostras de méis pela termogravimetria e que estas variáveis podem ser otimizadas independentemente, já que não foram observados efeitos de interação significativos entre elas. Em vista destes resultados pode-se afir- mar que cadinhos com maior volume minimizam o efeito de borbulhamento observado durante o aquecimento de alimentos com altas concentrações de açúcar e água, além de aumentar a interação da atmosfera oxidante com a amostra. Isto resulta em uma oxidação mais uniforme da matéria orgânica e, conseqüentemente, em menores teores de cinzas. O material do cadinho, por sua vez, influencia no processo de incineração somente devido às suas características de transferência de calor, resultando em teores de cinzas maiores ou menores para a amostra de mel, de acordo com o tipo de material utilizado. Antes de escolher o material de cadinho mais adequado para incineração de amostras deste alimento, é importante ressaltar que esta variável quando estudada em conjunto com outras, tais como taxa de aquecimento, massa de amostra, vazão de gás, pode produzir resultados diferentes dos encontrados aqui. Sendo assim, para se obter uma otimização completa do método termogravimétrico, a influência de outros fatores de natureza instrumental e relacionados às características da amostra, que podem interferir nos resultados, será investigada por outros planejamentos experimentais em trabalho futuro.

\section{REFERÊNCIAS}

1. Harwalkar, V. R.; MA, C-Y.; Thermal Analysis of Foods, $1^{\text {st }}$ ed., Elsevier Applied Science: Londres, 1990.

2. Haines, P. J.; Thermal Methods of Analysis: principles, applications and problems, $5^{\text {th }}$ ed., Chapman \& Hall: Oxford, 1995.

3. Wendlandt, W. W. M.; Thermal Analysis, $3^{\text {rd }}$ ed., John Wiley \& Sons: New York, 1986.

4. Felsner, M. L.; Matos, J. R.; An. Assoc. Bras. Quím. 1998, 47, 308.

5. Gennaro, L.; Bocca, A. P.; Modesti, D.; Masella, R.; Coni, E.; J. Agric. Food Chem. 1998, 46, 4465.

6. Tomassetti, M.; Campanella, L.; Aureli, T.; Thermochim. Acta 1989, 143, 15.

7. Acquistucci, R.; Bucci, R.; Magri, A. D.; Magri, A. L.; Thermochim. Acta 1991, 188,51 .

8. Tomassetti, M.; Campanella, L.; Aureli, T.; Sammartino, M. P.; Thermochim. Acta 1991, 190, 131.

9. Macêdo, R. O.; de Moura, O. M.; Souza, A. G.; Macêdo, A. M. C.; J. Therm. Anal. 1997, 49, 857.

10. Aggarwal, P.; Dollimore, D.; Talanta. 1999, 43, 1527.

11. Curini, R.; Dascenzo, G.; Materazzi, S.; Chiacchierini, E.; Curtis, S.D., Lucchetti, M.C.; Ann. Chim. 1991, 81, 625.

12. Nielsen, S. S.; Introduction to the Chemical Analysis of Foods, $1^{\text {st }}$ ed.; Jones and Bartlett Publishers: Boston, 1994.

13. Box, G. E. P.; Hunter, W. G.; Hunter, J. S.; Statistics for Experimenters, $1^{\text {st }}$ ed., Willey Press: New York, 1978.

14. Barros Neto, B. de; Scarminio, I. S.; Bruns, R. E.; Como fazer experimentos, $2^{\mathrm{a}}$ ed., Unicamp: Campinas, 2002.

15. Statsoft Incorporation; Statistica and Statistica Industrial System, Tulsa: USA, 1995.

16. Felsner, M.L.; Tese de Doutorado, Universidade de São Paulo, Brasil, 2001.

17. Brasil. Ministério da Agricultura e do Abastecimento. Instrução Normativa n. 11 , de 20 de outubro de 2000. Regulamento Técnico de Identidade e Qualidade de Mel. Diário Oficial da União, Brasília, n. 204, 23 out 2000, Seção 1, p. 16.

18. Codex Alimentarius Standard for Honey, Ref. Nr. CL 1993/14-SH FAO e WHO, Roma, 1993 